\title{
Instrumentación electrónica de una estación meteorológica automática
}

\section{Electronic instrumentation of an automatic weather station}

ROMERO-RODRÍGUEZ, Gabriel†*, HUESCA-LAZCANO, Erick Eduardo, FLORES-RAMÍREZ, Óscar y MARTÍNEZ-ÁNGELES, Jorge Rafael

Universidad Politécnica de Amozoc, Ingeniería Automotriz

ID $1^{\text {er }}$ Autor: Gabriel, Romero-Rodríguez / ORC ID: 0000-0002-5124-3016, Researcher ID Thomson: Y-2756-2018, CVU CONACYT ID: 299737

ID $1^{\text {er }}$ Coautor: Erick Eduardo, Huesca-Lazcano / ORC ID: 0000-0002-0505-8442, Researcher ID Thomson: F-1162-2018, CVU CONACYT ID: 223342

ID $2^{\text {do }}$ Coautor: Óscar, Flores-Ramírez / ORC ID: 0000-0001-9884-9499, Researcher ID Thomson: E-8242-2018, CVU CONACYT ID: 92914

ID $3^{\text {er }}$ Coautor: Jorge Rafael, Martínez-Ángeles / ORC ID: 0000-0003-1473-397X, Researcher ID Thomson: AAP-30082020

DOI: $10.35429 /$ JSI.2020.15.4.19.30

Recibido Julio 15, 2020; Aceptado Diciembre 30, 2020

\section{Resumen}

Los estudios climatológicos presentan una restricción común en la toma de decisiones basadas en datos meteorológicos, ya que los modelos climáticos frecuentemente carecen de valores precisos de sus parámetros en función del punto geográfico donde se adquiere la información, por lo que la adquisición climática en posiciones geográficas específicas resulta una posibilidad interesante. El objetivo del presente estudio fue desarrollar la instrumentación electrónica de una estación meteorológica automática (EMA) empleando un medio controlador con sensores que miden la temperatura del aire y del suelo $\left({ }^{\circ} \mathrm{C}\right)$, la humedad relativa del aire $(\%)$, la radiación solar global $(\mathrm{Wm} 2)$ y la velocidad del viento $(\mathrm{m} / \mathrm{s})$. Los datos adquiridos fueron comparados con estaciones comerciales Vantage Pro (VP) y GroWeather (GW) de la familia Davis Instruments donde se obtuvo un $\mathrm{R}^{2}$ de 0.93 en Temperatura y Humedad Relativa del aire y un $\mathrm{R}^{2}$ de 0.94 para la radiación solar. Así la EMA desarrollada presenta bondades en la calidad de los datos adquiridos que pueden ser usados para estudios de modelación matemática y generación de toma de decisiones.

Estación meteorológica automática (EMA), Monitoreo climático, Instrumentación electrónica

\begin{abstract}
Climatological studies present a common restriction in decisionmaking based on meteorological data, since climate models often lack precise values of their parameters depending on the geographical point where the information is acquired, therefore, climate acquisition in specific geographical positions is an interesting possibility. The objective of the present study was to develop the electronic instrumentation of an automatic weather station (AWS) using a controlling medium with sensors that measure air and soil temperature $\left({ }^{\circ} \mathrm{C}\right)$, relative humidity $(\%)$, solar radiation $\left(\mathrm{Wm}^{2}\right)$ and wind speed $(\mathrm{m} / \mathrm{s})$. The acquired data were compared with commercial stations Vantage Pro (VP) and GroWeather (GW) of the Davis Instruments family where an $\mathrm{R}^{2}$ of 0.93 was obtained in Temperature and Relative Humidity of the air and an $\mathrm{R}^{2}$ of 0.94 for solar radiation. Thus, the developed AWS presents benefits in the quality of the acquired data that can be used for studies of mathematical modeling and generation of decision-making.
\end{abstract}

Automatic weather station (AWS), Climate monitoring, Electronic instrumentation

Citación: ROMERO-RODRÍGUEZ, Gabriel, HUESCA-LAZCANO, Erick Eduardo, FLORES-RAMÍREZ, Óscar y MARTÍNEZ-ÁNGELES, Jorge Rafael. Instrumentación electrónica de una estación meteorológica automática. Revista de Innovación Sistemática. 2020. 4-15:19-30. 


\section{Introducción}

Una Estación Meteorológica Automática (EMA) es un equipo que integra diversos dispositivos con la capacidad de medir y almacenar variables climáticas relevantes a una necesidad determinada. El almacenamiento de la información se realiza a intervalos regulares, más tarde se procesa la información en gráficos y modelos que permiten realizar toma de decisiones (CAA, 2014).

Una exigencia en la tecnología empleada en las EMA radica en la cantidad, calidad y facilidad para registrar un gran número de variables climáticas, donde se debe considerar que, a mayor número de variables, flexibilidad de almacenamiento, procesamiento, captura y precisión de los datos, mayor será su costo económico.

La elección de una EMA dependerá de los requerimientos y objetivos que se han trazado el usuario. Para ello es relevante conocer y entender las características técnicas de las EMA, número y tipo de sensores para las variables a monitorear, compatibilidad de sensores y repuestos, tipos de almacenamiento y opciones de comunicación (Torres-Ruiz, 2006; CONAGUA, 2010).

Para comprender lo anterior, una EMA básica consta de dos unidades típicas: sensores y una tarjeta de adquisición de datos. Un sensor es un dispositivo que recibe un estímulo y lo transforma en una señal eléctrica, la cual se interpreta en una escala de medida acorde a la variable que se desea monitorear.

Una tarjeta de adquisición de datos es un dispositivo electrónico que envía y adquiere información de los sensores que están conectados a él; frecuentemente incorporan un microprocesador, una unidad de memoria para el almacenamiento y una fuente de energía para su funcionamiento. Las variables climáticas típicas para medir son: temperatura del aire, precipitación, humedad relativa, radiación solar, velocidad y dirección del viento (Medina-García et al., 2008).
Actualmente existen diversos sistemas de monitoreo de datos climáticos, los desarrollos actuales presentan visualización de datos e integración de sensores múltiples. Algunas de las EMA más usadas son: Vantage Pro de Davis, Onset de HOBO; con sistemas de adquisición de datos, sensores y comunicaciones con una plataforma multisensorial aplicada a la agricultura con diferentes tipos de comunicación; Kipp \& Zonen, con registradores de datos y sensores solares que abarcan el espectro desde el Ultravioleta (UV) hasta el Infrarrojo lejano (FIR) y atmosféricos; sistema táctico de observación meteorológica TacMet巴 MAWS201M de Vaisala, alto desempeño para diversas operaciones de campo; sistemas de adquisición de datos series CR-100-1000 de Campbell Scientific para precisión y rapidez de datos climáticos. Algunas EMA's de gama baja son: La Crosse Technology, Honeywell Technology Solutions, TFA; Sistema denominado MS-120-150 de la empresa DYACON se refiera a una estación meteorológica con múltiples sensores y sistema de comunicación embebido y software con aplicaciones predefinidas (Sivakumar et al., 2000; World Meteorological Organization, 2012; Rehman et al., 2014, Davis Instruments. 2020).

Una alternativa para obtener datos meteorológicos consiste en generar EMA's mediante el uso de dispositivos electrónicos de código abierto, los cuales suelen ser mucho más económicos que los dataloggers comerciales. Algunos de estos trabajos se mencionan a continuación.

Se han utilizado técnicas más nuevas que involucran telemetría satelital (TS) y sistemas de información geográfica (SIG) (Sivakumar, et. al, 2004; Al-Mahdi et. al, 2014). Para la recolección de los datos generados por cada estación se utilizó comunicación por medio de routers NanoStation, con un sistema de repetición entre los puntos o nodos (Pérez y Martínez, 2011). Garzón (2004) describe una red experimental que permite el estudio de las comunicaciones frente a condiciones climatológicas. Estas estaciones toman diferentes datos meteorológicos (presión, humedad, precipitaciones y temperatura). 
Este proyecto implemento una ESAP
(estación meteorológica para medidas pluviométricas) y se comunica con otras estaciones a través de redes de telefonía móvil (GSM) y radioenlaces. Meza (2007) desarrolló un proyecto que utiliza radares meteorológicos, controlados por el instituto INAMEH. Esta es una red de ocho radares meteorológicos los cuales son utilizados para estudios de precipitaciones, rastreo de tormentas y densidad del aire. Estos radares se encuentran enlazados en una red WLAN.

Los desarrollos anteriores presentan algunos problemas relacionados con la precisión de las mediciones, en la invención mencionada se presentan ventajas en las mediciones de temperatura del aire y suelo, humedad relativa del aire y suelo, y la radiación solar presenta mejor sensibilidad.

Los objetivos del presente trabajo consisten en hacer eficiente la adquisición y monitoreo de datos meteorológicos mediante mejora de la precisión en mediciones de variables climáticas como la temperatura del aire y de la masa térmica, humedad relativa del aire y la masa térmica, radiación solar y velocidad del viento; mediante el diseño y validación de una EMA la cual se denominará en lo siguiente como CLIMA.

\section{Metodología}

La EMA CLIMA se compone de distintos instrumentos electrónicos que la integran. A continuación, se describe la forma de interacción entre los dispositivos que la componen. En la Figura 1 se muestra el diagrama a bloques de la interacción general de los dispositivos electrónicos y sensores de la invención de medición y monitoreo de variables meteorológicas: el medio controlador (1), el suministro de voltaje (2), el medio de visualización (3), la unidad de almacenamiento de información (4), el medio contador de tiempo (5), el medio sensor de temperatura y humedad relativa del aire (6), el medio sensor de temperatura y humedad relativa del suelo (7), el medio sensor de la radiación solar (8) y el medio sensor de la velocidad del viento (9).

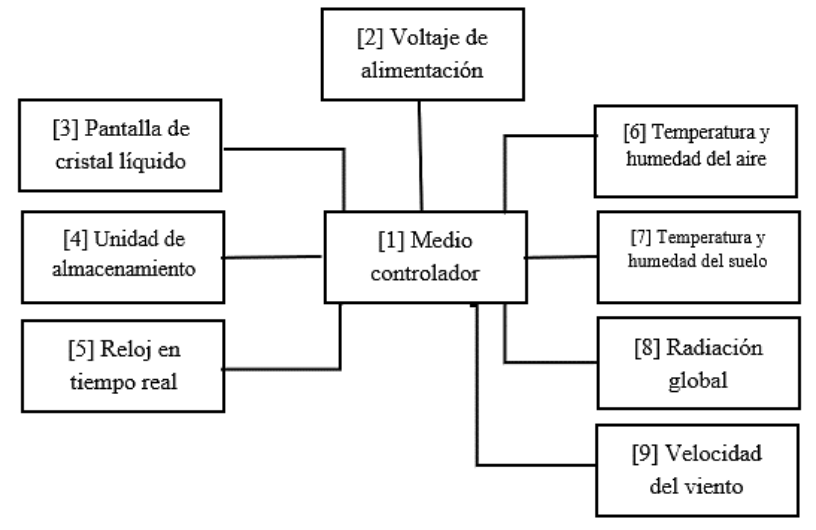

Figura 1 Interacción general de los dispositivos electrónicos y sensores de la EMA (CLIMA)

Fuente: Laboratorio de electrónica, Universidad Politécnica de Amozoc

El circuito electrónico de CLIMA se presenta en la Figura 2 y enseguida se explica la interacción de los dispositivos. El medio controlador cuenta con una arquitectura RISC a 8 bits, contiene $256 \mathrm{~Kb}$ de memoria flash para programación, $8 \mathrm{~KB}$ de RAM, cuenta con interfaz 2-wire, SPI y 4 UART, la velocidad de frecuencia es $16 \mathrm{MHz}$, contiene 54 puertos de entrada y salida programables de los cuales 14 puertos PWM, 16 canales de ADC de 10 bits, 6 temporizadores, su encapsulado es TQFP-100.

El medio controlador está conectado a una placa, la placa cuenta con una entrada para una fuente de alimentación externa y un regulador de voltaje, de manera que se pueden conectar de 5-12 VDC, cuenta con pines de alimentación de salida de $5 \mathrm{v}, 3.3 \mathrm{v}$ y voltaje de entrada de la fuente externa (Vin) además de sus tierras comunes GND, también cuenta con una interfaz de comunicación USB por los puertos UART y un botón de reset.

Para su mejor uso, el medio controlador está conectado a $12 \mathrm{v}$ en su fuente de alimentación externa, y los pines de comunicación $\mathrm{i}^{2} \mathrm{c}$ están conectados de la siguiente forma: el pin (20) del medio controlador se conecta a una resistencia de $10 \mathrm{~K} \Omega$ y esta a su vez se conecta a la alimentación común de $(5 \mathrm{v})$, el pin (21) del medio controlador se conecta a una resistencia de $10 \mathrm{~K} \Omega$ y esta a su vez se conecta a la alimentación común de $(5 \mathrm{v})$. El sensor que mide las variaciones de temperatura $(\mathrm{T})$ del aire en grados Celsius o Fahrenheit, así como la humedad relativa (HR) en porcentaje (\%). 
Este dispositivo tiene 4 terminales, las terminales (1) se conectan al suministro de 5 volts DC y la terminal 4 a tierra (GND) común, la comunicación del dispositivo se hace de forma serial y bidireccional $\left(\mathrm{i}^{2} \mathrm{c}\right)$ por lo que la salida de datos que está en la terminal 2 se conecta al medio controlador en el pin (20 SDA) y la terminal 3 que corresponde al reloj serial se conecta en el pin (21) del medio controlador.

La unidad de almacenamiento de información es un dispositivo de memoria flash para tarjeta Secure Digital (SD), consta de 6 pines. Esta unidad se conecta con el medio controlador de la siguiente forma: el pin (1) de la memoria se conecta a (GND) común, el pin (2) de la memoria se conecta a alimentación (3.3V), el pin (3) de la unidad corresponde al Chip Select y se conecta al pin (53) del medio controlador. El pin (4) de la unidad corresponde a (MOSI) Salida-Maestro, Entrada-Esclavo; y se conecta al pin (51) del medio controlador; el pin (5) de la unidad es la señal del reloj del bus de datos SCK y se conecta al pin (52) del medio controlador; finalmente el pin (6) de la unidad es (MISO) Entrada-Maestro, Salida-Esclavo, se conecta al pin (50) del medio controlador.

El reloj en tiempo real consta de 4 pines para comunicarse con el medio controlador, además de una batería externa de $5 \mathrm{v}$ que permite que no se borre la información de la fecha y hora del reloj. Este dispositivo se conecta de la siguiente manera con el medio controlador: el pin (1) del reloj se conecta a tierra común (GND), el pin (2) del reloj se conecta a alimentación común (5v), el pin (3) se corresponde a la línea de datos (SDA) y se conecta al pin (20) del medio controlador; y el pin (4) del dispositivo es la línea del reloj (SCL) y se conecta al pin (21) del medio controlador.

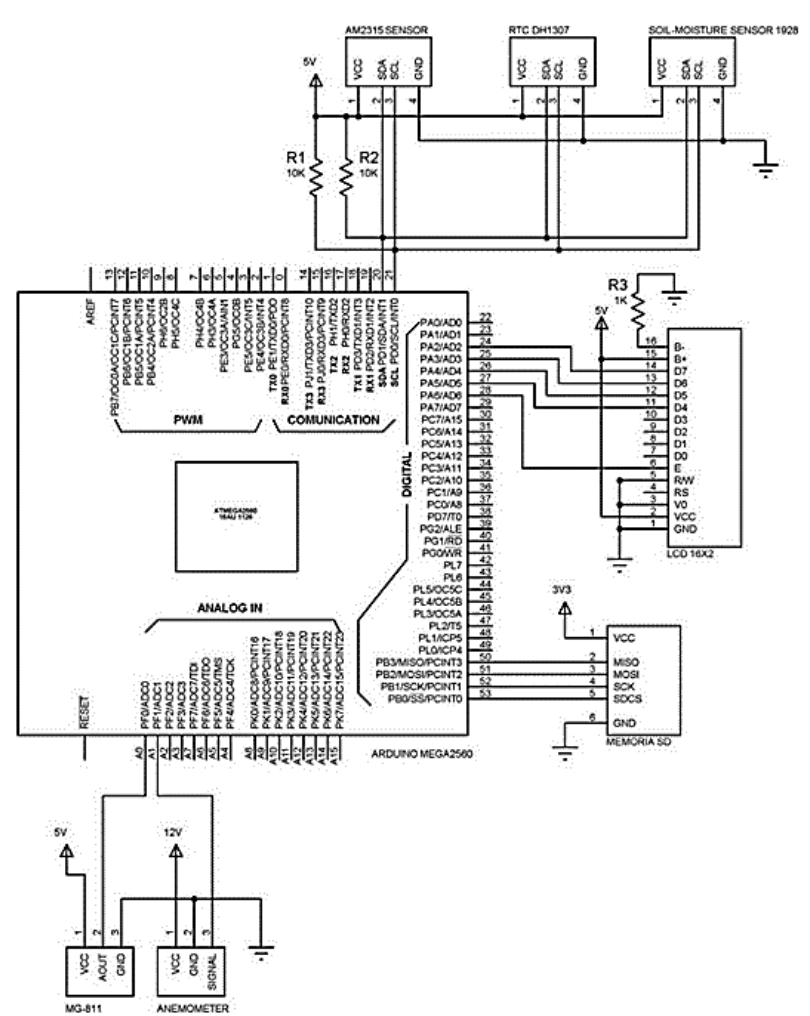

Figura 2 Diagrama esquemático del circuito electrónico de interconexión de la EMA (CLIMA)

Fuente: Laboratorio de electrónica, Universidad Politécnica de Amozoc

La pantalla de cristal líquido (LCD) es una pantalla delgada y plana formada por 16 columnas y 2 filas, con pixeles en color colocados delante de una fuente de luz azul. Este dispositivo fue usado ya que utiliza poca energía eléctrica $(5 \mathrm{v})$. Se conecta con el medio controlador de la siguiente forma: los pines $(1,3$, 5 y 16) del LCD se conectan a tierra común (GND), los pines ( 2 y 15 ) del LCD se conectan a alimentación común (5V) y el pin (4) del LCD se conecta al pin (7) del medio controlador.

El sensor SHT10 contiene dos módulos medir las variaciones de la masa térmica (suelo): el primero para la temperatura y otro para la humedad relativa, el dispositivo se protege de la interacción directa del suelo gracias a una malla metálica protectora. Este empaque metálico es a prueba de agua y evita que la humedad dañe al sensor, aunque permite un flujo constante de aire para medir la humedad del suelo. 
El sensor se conecta con el medio controlador de la siguiente forma: el pin (1) del sensor se conecta a alimentación común $(5 \mathrm{v})$, el pin (2) del sensor se conecta a tierra común (GND), el pin (4) del sensor es la señal de reloj SCK y se conecta al pin (21) del medio controlador, el pin (4) del sensor es la línea de datos (DATA) y se conecta al pin (20) del medio controlador.

El anemómetro es un instrumento para medir la velocidad de circulación de un fluido gaseoso, en especial del viento, funciona con el principio del efecto Hall para medir los cambios repentinos de la velocidad del viento, especialmente en las turbulencias, a través de un filamento caliente, que consiste en un hilo de platino calentado eléctricamente: la acción del viento tiene por efecto enfriarlo y hace variar así su resistencia; por consiguiente, la corriente que atraviesa el hilo es proporcional a la velocidad del viento. Se conecta al medio controlador de la siguiente forma: el pin (1) del anemómetro se alimenta a $12 \mathrm{~V}$, corresponde a (Vin) del medio controlador; el pin (2) del anemómetro se conecta a la tierra común (GND), y el pin (3) del anemómetro se conecta a la entrada analógica (A1) del medio controlador.

El piranómetro es un sensor de Radiación Solar Global es un sensor diseñado para medir la densidad del flujo de radiación solar (kilovatios por metro cuadrado) en un campo de 180 grados. Se constituye por una pila termoeléctrica contenida en un alojamiento con dos semiesferas de cristal. La pila termoeléctrica está constituida por una serie de termopares colocados horizontalmente, cuyos extremos están soldados con unas barras de cobre verticales solidarias a una placa de latón maciza. El conjunto está pintado con un barniz negro, para absorber la radiación. El flujo de calor originado por la radiación se transmite a la termopila, generándose una tensión eléctrica proporcional a la diferencia de temperatura entre los metales de los termopares. El piranómetro se conecta con el medio controlador de la siguiente manera: el pin (1) del piranómetro se conecta a tierra común (GND), el pin (2) del piranómetro se alimenta a (5V) y el pin (3) del piranómetro se conecta a la entrada analógica del medio controlador (A0); en la Tabla 1 se describen los instrumentos usados.
La forma en que se comunica el medio controlador con los dispositivos electrónicos lleva los pasos lógicos que se presentan en el diagrama de la Figura 3. No obstante que la anterior descripción se realizó tomando en cuenta las modalidades preferidas de CLIMA, en sentido amplio y no limitativo. El entorno de software para la programación de la EMA se basó en el sistema operativo Windows, utilizando IDE para programar en un lenguaje basado en $\mathrm{C}++$ (Wiring + Processing).

\begin{tabular}{|c|c|c|}
\hline Variable & Sensor & Características \\
\hline $\begin{array}{l}\text { Temperatura } \\
\text { y Humedad } \\
\text { del aire }\end{array}$ & $\begin{array}{l}\text { AOSONG } \\
\text { AM2315 }\end{array}$ & $\begin{array}{l}\text { Resolución: } 16 \text { bits } \\
\text { Histéresis: } \pm 0.1 \% \text { HR y } \pm \\
0.2^{\circ} \mathrm{C} \\
\text { Precisión de humedad: } \pm \\
2 \% \mathrm{HR} \text { a } 25^{\circ} \mathrm{C} \\
\text { Precisión de temp: } \pm \\
0.12^{\circ} \mathrm{C} \text { a } 25^{\circ} \mathrm{C} \\
\text { Voltaje de alimentación: } \\
\text { DC } 3.5 \sim 5.5 \mathrm{~V}\end{array}$ \\
\hline $\begin{array}{l}\text { Temperatura } \\
\text { y Humedad } \\
\text { del suelo }\end{array}$ & $\begin{array}{l}\text { SONBEST } \\
\text { SHT10 }\end{array}$ & $\begin{array}{l}\text { Rango de temp: }-40 \\
+123.8^{\circ} \mathrm{C} \\
\text { Precisión de temp: } \pm 0.3- \\
0.5^{\circ} \mathrm{C} \text { a } 25^{\circ} \mathrm{C} \\
\text { Rango de humedad: } 0- \\
100 \% \\
\text { Precisión de humedad: } \\
\pm 1.8-4.5 \% \mathrm{HR} \\
\text { Voltaje de alimentación: } \\
\text { DC } 3.5 \sim 5 \mathrm{~V}\end{array}$ \\
\hline $\begin{array}{l}\text { Radiación } \\
\text { Global } \\
\left(\mathrm{Wm}^{2}\right)\end{array}$ & $\begin{array}{l}\text { KOSMOS } \\
\text { SP03670I }\end{array}$ & $\begin{array}{l}\text { Rango: } 1-1250 \mathrm{w} / \mathrm{m} 2+-5 \% \\
\text { Voltaje de Excitación: } 3.0 \text { a } \\
5.0 \text { VDC } \\
\text { Salida del Sensor: } 0 \text { a } 2.5 \mathrm{~V} \\
\text { Linear: W/m2 = V*500 } \\
\text { Corrimiento: } 1 \% \text { por año } \\
\text { incluye: nivel de burbuja } \\
\text { Precisión: }+/-5 \% \text {. }\end{array}$ \\
\hline $\begin{array}{l}\text { Velocidad de } \\
\text { Viento }\left(\mathrm{ms}^{-1}\right)\end{array}$ & $\begin{array}{l}\text { ADAFRUIT } \\
1733\end{array}$ & $\begin{array}{l}\text { Voltaje de alimentación: } \\
\text { DC } 12 \mathrm{~V} \\
\text { Salida de voltaje: } 0.4 \text { a } 2 \mathrm{~V} \\
\text { DC } \\
\text { Rango de prueba: } 0.5 \mathrm{~m} / \mathrm{s} \text { a } \\
50 \mathrm{~m} / \mathrm{s} \\
\text { Velocidad de viento inicial: } \\
0.2 \mathrm{~m} / \mathrm{s} \\
\text { Resolución: } 0.1 \mathrm{~m} / \mathrm{s} \\
\text { Precisión: en el peor caso } \\
1 \mathrm{~m} / \mathrm{s} \\
\text { Máxima vel. de viento: } \\
70 \mathrm{~m} / \mathrm{s}\end{array}$ \\
\hline
\end{tabular}

Tabla 1 Variables climáticas medidas en el invernadero, tipo de sensores y características

Fuente: Aosong, Sonbest, Parallax, Kosmos y Adafruit

Una parte importante en la aportación de la EMA CLIMA diseñada radica en la programación que optimiza la adquisición y almacenamiento de los datos de las variables medidas y amplia las opciones de comunicación y manejo de la información. 
La Figura 12 muestra el diagrama de flujo principal de la invención, que integra los pasos lógicos de comunicación entre el medio controlador y los dispositivos para censar mediante la inicialización de los componentes, medición del tiempo, almacenamiento de información, medio para desplegar la información y el monitoreo que establece la forma en que presenta la información adquirida.

La inicialización de los elementos que integran la EMA depende de la comunicación que existe entre el medio controlador y sus elementos, en caso de no haber comunicación se genera un error de arranque y en caso contrario se inicia la conexión con el medio controlador y se verifican uno a uno los elementos que integran. Para el reloj se verifica la conexión entre el dispositivo y el medio controlador en caso de no haber conexión se despliega en el LCD un mensaje de falla, en caso contrario se inicializa el reloj y se muestra un mensaje de funcionamiento correcto. Para la unidad de almacenamiento de la información SD se verifica la conexión en caso de no haber conexión se despliega en el LCD un mensaje de falla, en caso contrario se inicializa la SD y se muestra un mensaje de funcionamiento correcto.

Para el sensor de temperatura del aire se verifica la conexión en caso de no haber conexión se despliega un mensaje de falla, en caso contrario se inicializa el sensor y se muestra un mensaje de funcionamiento correcto. Para el sensor de temperatura del suelo se verifica la conexión en caso de no haber conexión se despliega un mensaje de falla, en caso contrario se inicializa el sensor y se muestra un mensaje de funcionamiento correcto. Para el sensor de humedad relativa del aire se verifica la conexión en caso de no haber conexión se despliega un mensaje de falla, en caso contrario se inicializa el sensor $y$ se muestra un mensaje de funcionamiento correcto.

Para el sensor de humedad relativa del suelo se verifica la conexión en caso de no haber conexión se despliega un mensaje de falla, en caso contrario se inicializa el sensor y se muestra un mensaje de funcionamiento correcto. El anemómetro verifica su conexión en caso de no haber conexión se despliega un mensaje de falla, en caso contrario se inicializa el sensor y se muestra un mensaje de funcionamiento correcto.
El piranómetro verifica su conexión en caso de no haber conexión se despliega un mensaje de falla, en caso contrario se inicializa el sensor $y$ se muestra un mensaje de funcionamiento correcto.

Finalmente, el LCD muestra un mensaje de bienvenida y la SD abre un archivo para almacenar información y el siguiente paso lógico es continuar con el proceso de monitoreo.

El flujo de información que sigue el monitoreo, una vez iniciados los componentes, prioriza el funcionamiento correcto del reloj y en caso de no haber funcionamiento envía un error, en caso contrario adquiere la fecha y el tiempo, luego tiene dos opciones principales: la adquisición y el monitoreo. Por un lado, la adquisición considera que cada cinco minutos la SD adquiera el tiempo y la fecha, la temperatura del aire y del suelo, la humedad relativa del aire y del suelo, la radiación solar y la velocidad del viento.

Por otro lado el monitoreo emplea interrupciones de tiempo para mostrar en el LCD la información adquirida de los sensores; la primer interrupción permite mostrar la fecha, hora, temperatura y humedad relativa del aire, la segunda interrupción permite mostrar la fecha, hora, temperatura y humedad relativa del suelo, la tercer interrupción permite mostrar la fecha, hora y velocidad del viento, la cuarta interrupción permite mostrar la fecha, hora y radiación solar, luego se reinician las interrupciones para repetir el ciclo, finalmente si se abandona el proceso de monitoreo la lógica direcciona la finalización del proceso.

La forma en que la unidad de almacenamiento SD procesa la información es: al inicio verifica que exista comunicación con el medio controlador en caso de no haber comunicación se envía un error, en caso contrario se realizan dos procesos síncronos: la recepción y la transmisión.

Por un lado, la recepción considera el proceso de lectura de datos provenientes del medio controlador en caso de no haber lectura revisa si existe comunicación, en caso contrario inicia la lectura de la información: fecha y tiempo, temperatura aire y suelo, humedad relativa aire y suelo, velocidad de viento $y$ radiación solar. 
La transmisión considera el proceso de escritura donde una vez abierto el archivo de generación de texto (datos.txt) realiza la lectura del tiempo inicial, en caso de que coincida con el tiempo final significa que ha avanzado cinco minutos y esto le permite realizar el almacenamiento el cual considera el espacio de memoria inicial y procede a almacenar la información que ha leído del medio controlador: fecha y tiempo, temperatura aire y suelo, humedad relativa aire y suelo, velocidad de viento y radiación solar; luego incrementa el espacio de memoria y regresa a verificar el avance del tiempo. El reloj en tiempo real realiza los pasos lógicos para adquirir fecha y tiempo como sigue: primero verifica si existe comunicación, en caso de que no haya comunicación se envía un error, en caso contrario realiza la adquisición del tiempo y fecha. Así, el tiempo adquiere la hora entre los números cero y veintitrés, después adquiere los minutos entre cero y cincuenta y nueve y para los segundos entre cero y cincuenta y nueve, después de adquirir los tres datos envía la información de tiempo. Después, la adquisición de la fecha primero verifica el día que puede seleccionarse entre los días de la semana, después verifica el mes y selecciona entre los meses del año, por último, se ajusta el año y se envía la información de fecha y se finaliza el ciclo.

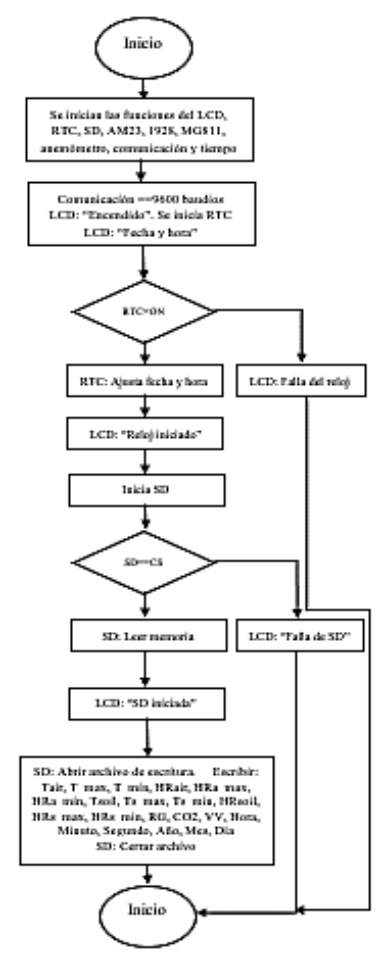

Figura 3 Flujo de los pasos lógicos de comunicación del medio controlador con los dispositivos electrónicos

Fuente: Laboratorio de electrónica, Universidad Politécnica de Amozoc
Los pasos lógicos que sigue el medio de visualización de la información LCD verifica que haya comunicación con el medio controlador, en caso de que no haya comunicación envía un error, en caso contrario realiza dos procesos: inicialización y monitoreo.

Primero, la inicialización muestra un mensaje de bienvenida al usuario; después, el monitoreo despliega los datos adquiridos en el medio controlador, cuya visualización depende de las interrupciones y puede seleccionar entre fecha, tiempo, temperatura y humedad relativa del aire, fecha, tiempo, temperatura y humedad relativa del suelo, fecha, tiempo y velocidad del viento $\mathrm{y}$, fecha, tiempo y radiación solar, después de ello finaliza el proceso.

Los pasos que sigue la comunicación del medio controlador con los dispositivos que conforman la EMA son: primero verifica que el buffer de comunicación esté habilitado en caso de no estar habilitado manda un error, en caso contrario verifica el tamaño de las palabras digitales y genera los espacios de memoria a usar, después verifica que exista transmisión, en caso no haber transmisión verifica el estado del buffer, en caso contrario para iniciar verifica la velocidad de transmisión, ajusta el reloj, habilita proceso de lectura y escritura de las interrupciones; finalmente el proceso continua con el proceso de inicialización.

La lógica que persigue el error es: siempre verifica la alimentación de energía y en caso de haber alimentación verifica la comunicación y en caso contrario termina el proceso (se debe entender que el medio controlador no tiene energía para funcionar). 


\section{Resultados}

Ya que la medición de datos climáticos representa un problema intrínsecamente relacionado con el comportamiento del medio, la calidad de los instrumentos para medir, almacenar y representar la información; la solución que presenta la EMA CLIMA es la adquisición de datos climáticos con precisión, esto significa que las variables climáticas: temperatura del aire y del suelo, humedad relativa del aire y del suelo, radiación solar y velocidad del viento, fueron medidas y validadas en comparación con otros equipos climáticos; puestos a prueba en el Colegio de Postgraduados, Campus Montecillo, México durante 15 días contra las estaciones comerciales: Vantage Pro (VP) y GroWeather (GW) Figura 4, donde se encontraron características descritas a continuación.

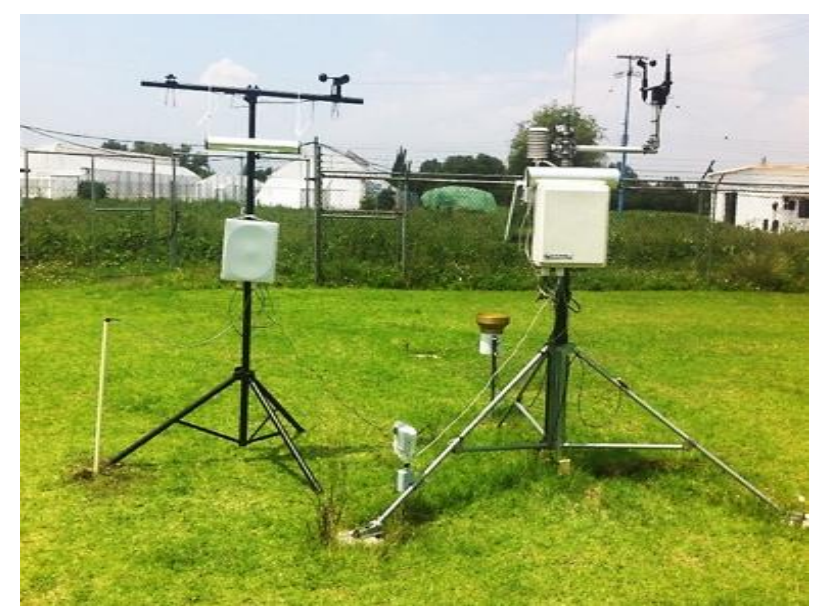

Figura 4 Comparación de desempeño en la adquisición de datos de la EMA CLIMA contra Vantage Pro y GroWather de Davis Instruments

Fuente: UPAM-COLPOS Montecillo

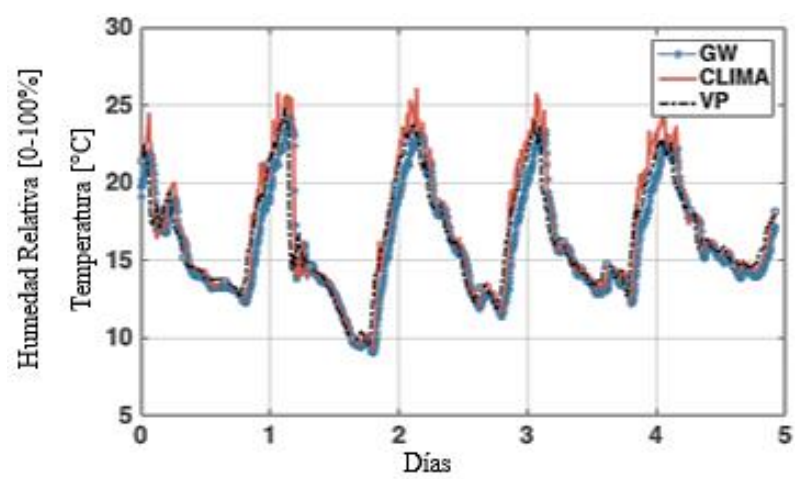

Figura 5 Desempeño de la temperatura del aire durante 5 días en comparación de CLIMA contra Vantage Pro y GroWather

Fuente: Universidad Politécnica de Amozoc

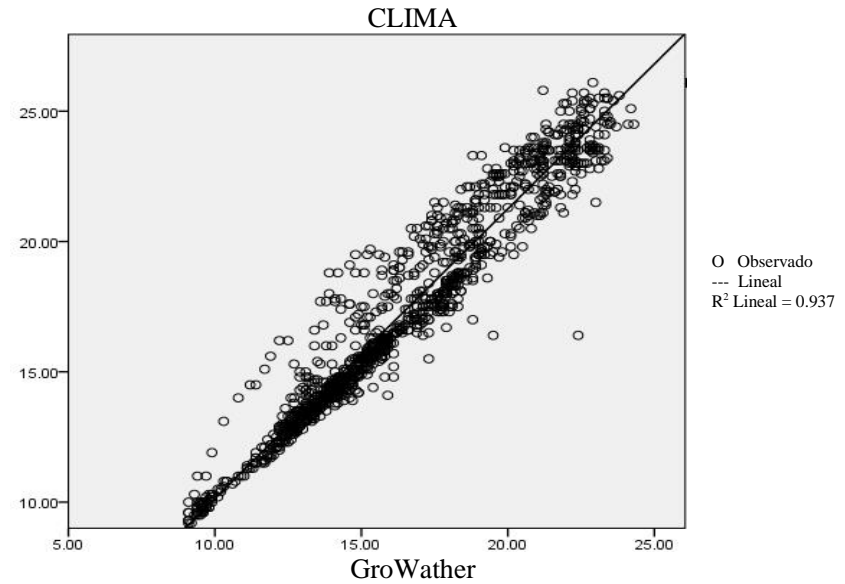

Figura 6 Regresión cuadrática lineal $\left(\mathrm{R}^{2}\right)$ de la temperatura del aire CLIMA vs GW

Fuente: Universidad Politécnica de Amozoc

La temperatura de aire (Figura 5) con respecto a las estaciones comerciales, CLIMA presenta un buen desempeño, teniendo diferencias acentuadas en los puntos más altos de temperatura, dado que la precisión y sensibilidad del sensor supera las características de los sensores de dichas estaciones.

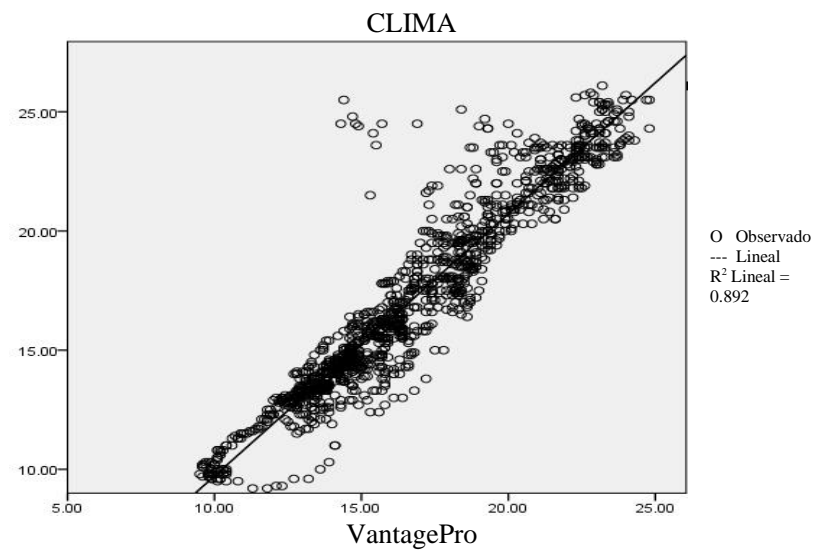

Figura 7 Regresión cuadrática lineal $\left(\mathrm{R}^{2}\right)$ de la temperatura del aire CLIMA vs VP

Fuente: Universidad Politécnica de Amozoc

Como parte de la comparación en la precisión de los datos medidos, se realizó una regresión lineal (Figura 6 y 7) para determinar la calidad del equipo desarrollado y su capacidad para replicar las magnitudes de los datos medidos usando el coeficiente de determinación $\mathrm{R}^{2}$. 


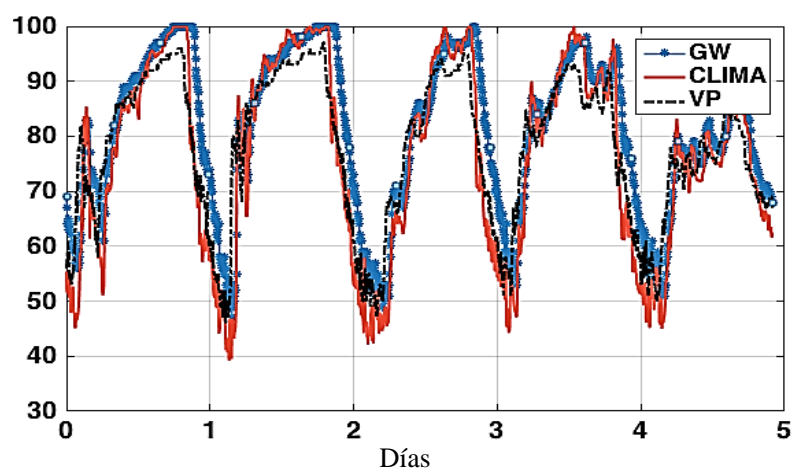

Figura 7 Desempeño de la humedad relativa de CLIMA contra Vantage Pro y GroWather

Fuente: Universidad Politécnica de Amozoc

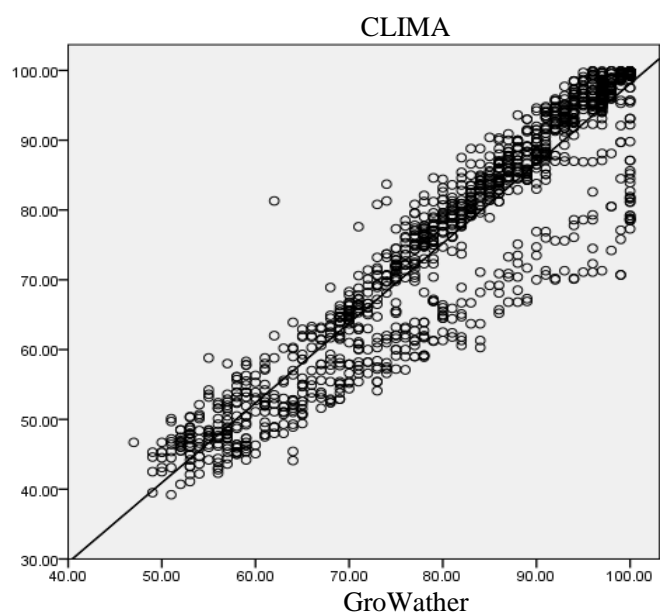

O Observado
R- Lineal
$\mathrm{R}^{2}$ Lineal $=0.88$

Figura 8 Regresión lineal cuadrática $\left(\mathrm{R}^{2}\right)$ de la humedad relativa CLIMA vs GW

Fuente: Universidad Politécnica de Amozoc

La humedad relativa de aire (Figura 8) con respecto a las estaciones comerciales presenta un buen comportamiento, teniendo diferencias acentuadas en los registros más altos y bajos de la humedad, dado que la precisión y sensibilidad del sensor supera las características de los sensores de dichas estaciones.

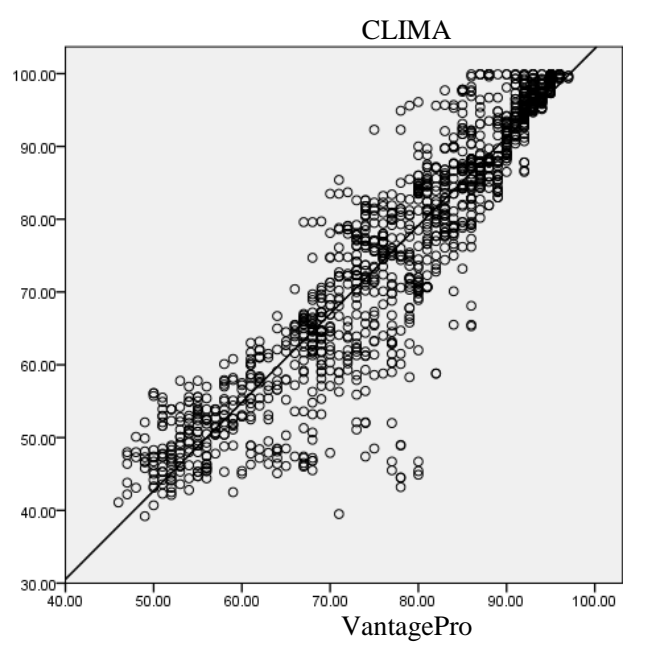

O Observado
--. Lineal
$\mathrm{R}^{2}$ Linal $\mathrm{R}^{2}$ Lineal $=0.859$

Figura 9 Regresión cuadrática lineal $\left(\mathrm{R}^{2}\right)$ de la humedad relativa CLIMA vs VP

Fuente: Universidad Politécnica de Amozoc
Las figuras 8 y 9 muestran las regresiones lineales entre la invención y $\mathrm{GW}$, y la invención y VP respectivamente.

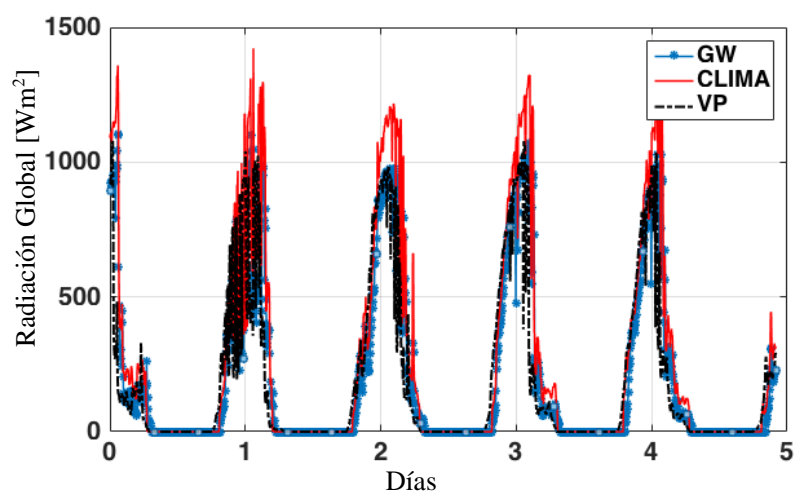

Figura 9 Comparación de las mediciones de radiación solar global entre CLIMA, GW y VP

Fuente: Universidad Politécnica de Amozoc

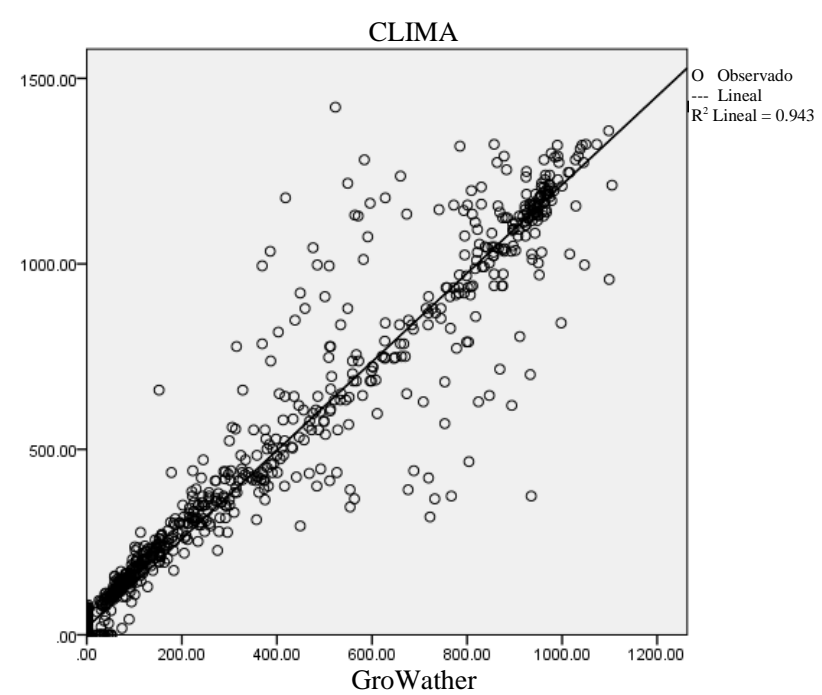

Figura 10 Regresión lineal cuadrática $\left(\mathrm{R}^{2}\right)$ de la radiación solar global CLIMA vs GW

Fuente: Universidad Politécnica de Amozoc

Las mediciones de la radiación solar global se muestran en la Figura 9, donde se observa que las mediciones realizadas por la EMA CLIMA presentan mayor sensibilidad a las variaciones que en ocasiones alcanzan los 1200 $\mathrm{Wm}^{2}$. 


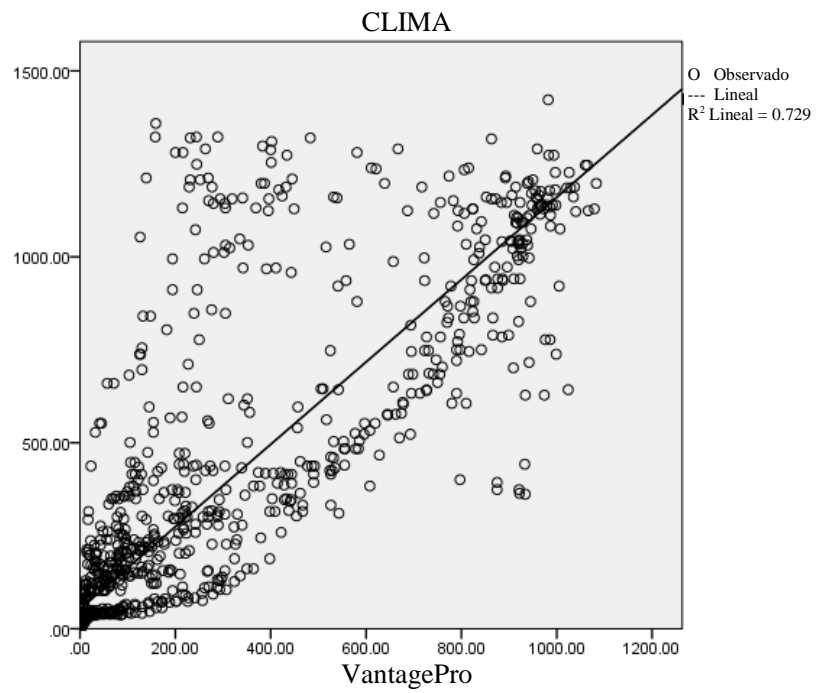

Figura 11 Regresión cuadrada $\left(\mathrm{R}^{2}\right)$ de la radiación solar global CLIMA vs VP

Fuente: Universidad Politécnica de Amozoc

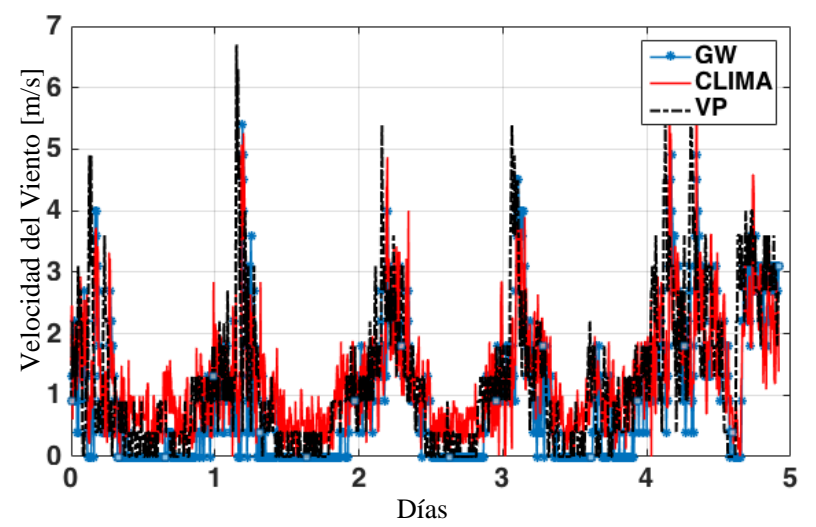

Figura 12 Desempeño de la velocidad de viento entre CLIMA, GW y VP

Fuente: Universidad Politécnica de Amozoc

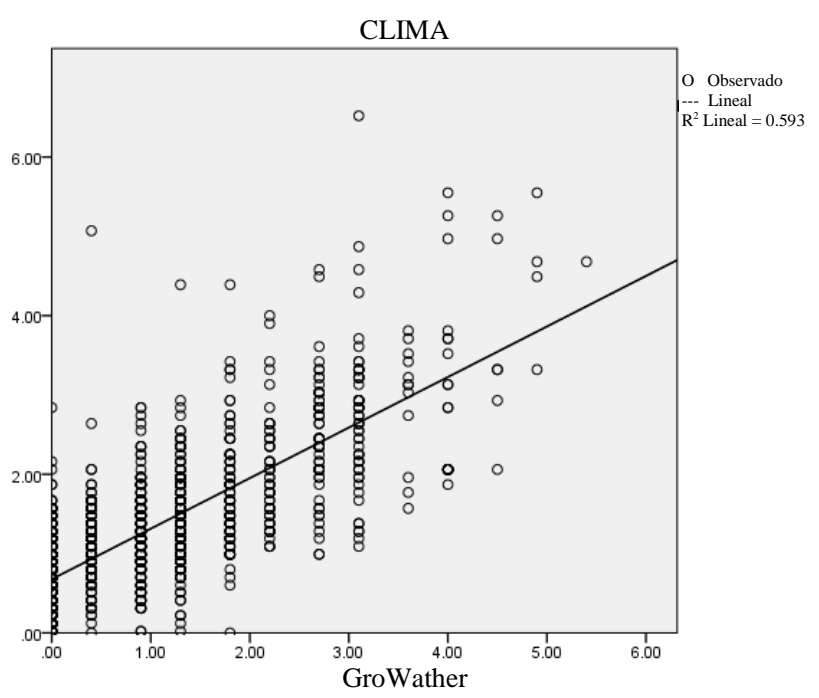

Figura 13 Regresión cuadrática $\left(\mathrm{R}^{2}\right)$ de la velocidad de viento CLIMA vs GW

Fuente: Universidad Politécnica de Amozoc

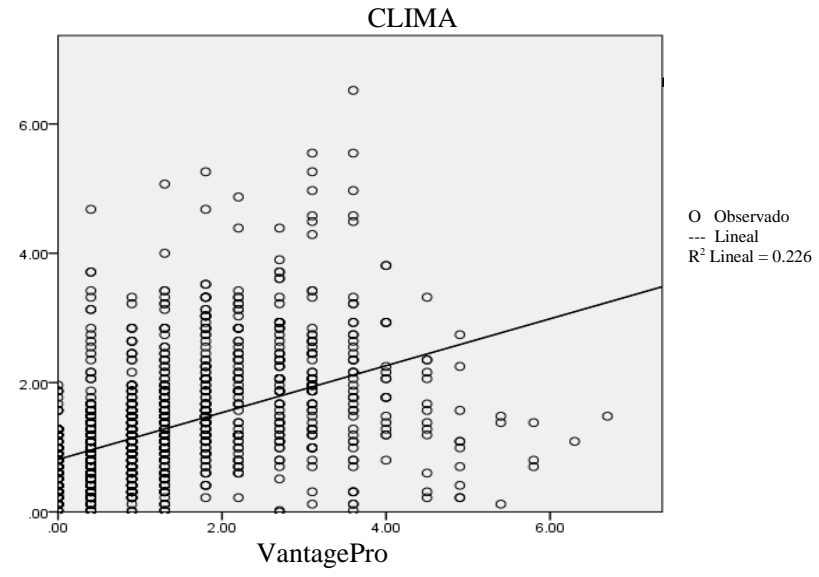

Figura 14 Regresión cuadrada $\left(\mathrm{R}^{2}\right)$ de la velocidad de viento CLIMA vs VP

Fuente: Universidad Politécnica de Amozoc

Las figuras 10 y 11 muestran las regresiones lineales entre la invención CLIMA y GW, y la invención CLIMA y VP respectivamente. La velocidad de viento (Figura 12) presenta el desempeño de CLIMA en comparación con las estaciones comerciales.

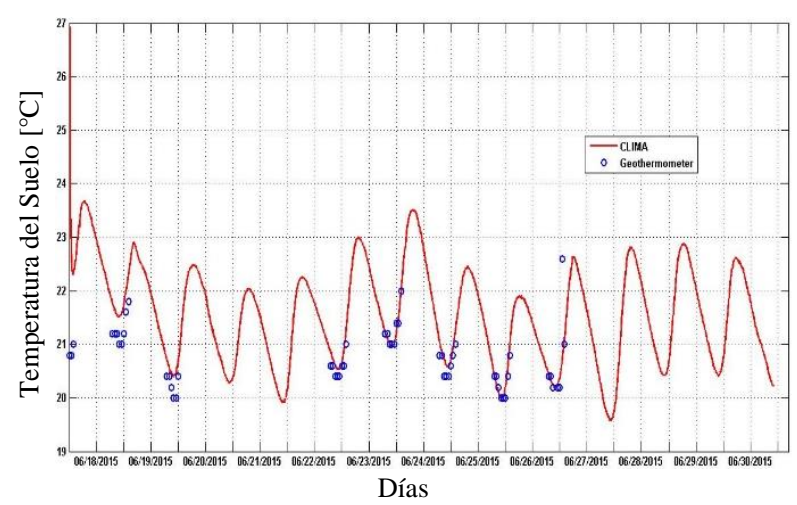

Figura 15 Desempeño de la temperatura del suelo entre CLIMA y geotermómetro analógico

Fuente: Universidad Politécnica de Amozoc

Las figuras 13 y 14 muestran las regresiones lineales entre la invención y GW, y la invención y VP respectivamente.

La temperatura y humedad relativa del suelo (Figura 15) no pudo compararse en su totalidad con las estaciones comerciales, dado que dichas estaciones no contemplan estas variables, pero se comparó parcialmente con geotermómetro analógico. 
Las ventajas técnicas que presenta CLIMA como sistema de adquisición de datos climáticos se resumen de forma numérica con los datos medidos y los valores estadísticos de $\mathrm{R}^{2}$ para cada variable que se muestran en la Tabla 2 donde se puede observar que la variable que presenta mejor precisión es la temperatura y la más lejana es la velocidad del viento.

\begin{tabular}{|l|r|r|}
\hline Variable & $\mathbf{R}^{2}$ Clima-GW & $\mathbf{R}^{2}$ Clima - VP \\
\hline T & 0.937 & 0.892 \\
\hline HR & 0.883 & 0.859 \\
\hline RG & 0.943 & 0.729 \\
\hline VV & 0.593 & 0.226 \\
\hline
\end{tabular}

Tabla 2 Valores de las regresiones cuadráticas lineales para cada variable y equipo comparado

Fuente: Universidad Politécnica de Amozoc

\section{Discusión de resultados}

Para realizar el proceso de validación, se tomó en cuenta que las estaciones de referencia Vantage Pro y Gro Weather del Colegio de Postgraduados Campus Montecillo se dispusieron $150 \mathrm{~cm}$ de distancia del prototipo CLIMA, los campos experimentales tuvieron las mismas condiciones, y los sensores presentaron las mismas características de precisión. Las alturas de los sensores fueron las mismas que las del prototipo. La separación entre las estaciones de referencia y CLIMA no pudo representar un factor que influyera en la comparación de los históricos. De acuerdo con Camargo y Hubbard (1999), para una distancia de $30 \mathrm{~km}$, en condiciones de terreno similar, hay $90 \%$ de probabilidades de que la variable sea igual.

La FAO (Allen et al., 2006) propuso una técnica para utilizar los datos de una estación cercana para suplir datos perdidos en otra. Los criterios que establecen para determinar si es viable utilizar estos datos como reemplazo es que, de una regresión lineal entre una serie de datos conocidos, del mismo periodo, de las dos estaciones meteorológicas, la $\beta$ esté entre 0.7 y 1.3 .

Así mismo, el coeficiente de determinación R2 debe ser mayor a 0.7. La $\beta$ y el coeficiente $\mathrm{R}^{2}$ obtenidos para todas las variables medidas (a excepción de la velocidad de viento) se encuentran dentro de los criterios establecidos por la FAO, por lo que se puede decir que estaciones están midiendo valores similares, lo que puede hacer que sea válido reemplazar los datos de una con otra.
Para el viento, aunque están dentro del rango de los $10 \mathrm{~km}$ aceptables para que la variable sea igual (Camargo y Hubbard, 1999), ni la correlación de la magnitud, ni la de dirección, alcanzan el criterio de 0.7 , por lo que la regresión lineal no es óptima.

\section{Conclusiones}

CLIMA soluciona la problemática de la medición y adquisición de las variables climáticas a bajo costo económico con precisión de hasta el $93 \%$ en temperatura, $88 \%$ en humedad relativa del aire, temperatura y humedad relativa del suelo, y hasta un $94 \%$ en radiación solar global, mientras que para la velocidad de viento presenta deficiencias que se encuentran en el producto comparado con las estaciones meteorológicas comerciales Vantage Pro y GroWeather radica en la medición de la velocidad de viento, ya que su regresión lineal $\left(\mathrm{R}^{2}\right)$ está por debajo de lo esperado, debido a las características intrínsecas del sensor cuyo principio es el efecto Hall, de manera que se puede modificar en un futuro y disponer un dispositivo de mayor precisión.

\section{Referencias}

Adafruit. 2020. [Consulta 23 de febrero de 2020] Disponible en: https://www.adafruit.com/

Aosong Electronics, Co. Ltd. Digital temperature and humidity sensor AM2315. Product Manual.

Allen, R. G.; Pereira, L. S.; Raes, D. y Smith, M. 2006. Evapotranspiración del cultivo. Traducción al español. Food and Agriculture Organization of the United Nations. Roma, Italia. $342 \mathrm{p}$.

Al-Mahdi, A. M.; Ndahi, E. M. S.; Yahaya, B. and Maina, M. L. 2014. Integrated gis and satellite remote sensing in mapping the growth, managing and production of inland water fisheries and aquaculture. Eur. Sci. J. 6(10):178183.

Arduino. 2020. An Open-Source Electronics Prototyping Platform. [Consulta 23 de febrero de 2020] Disponible en: http://www.arduino.cc 
Camargo, M. B. and Hubbard, K. G. 1999. Spatial and temporal variability of daily weather variables in sub-humid and semi-arid areas of the united states high plains. Agric. Forest Meteorol. (93):141-148.

Centro del Agua para la Agricultura (CAA), Universidad de Concepción, 2014. Recuperado de:http://files.dlhingenieria.webnode.cl/200000 087 -

12c7113c15/ESTACI\%C3\%93N\%20METERE OLOGICA\%20AUTOMATICA.pdf 4 de noviembre de 2019.

CONAGUA (Comisión Nacional del Agua). 2010. Proyecto de Modernización del Servicio Meteorológico Nacional de México: Diagnóstico Institucional y Propuesta de Plan Estratégico 2010-2019. 67 p.

Davis Instruments. 2020. Wireless Vantage Pro2 Plus. Recuperado de http://www.davisnet.com/weather/products/wea ther_product.asp?pnum $=06162$.

Fisher, D., K. and Gould, P., J. Open-Source Hardware Is a Low-Cost Alternative for Scientific Instrumentation and Research. SciRes. Modern Instrumentation, 2012, 1, 8-20. 2012.

Garzón A. Meteorológica y climatología semana de la ciencia y tecnología 2004, Fundación Española para las ciencias y la tecnología, España, 2004.

Kosmos. 2020. [Consulta 07 de marzo de 2020] Disponible en: http://www.kosmos.com.mx/

Medina-García, G.; Grageda- Grageda, J.; RuizCorral, J. A. and Báez- González A. D. 2008. Uso de estaciones meteorológicas en la agricultura. México. INIFAP.

Meza B., Vargas E. Propuesta de diseño de un sistema de comunicaciones alterno para la interconexión del sistema de radares meteorológicos a la sede del Inameh, Universidad Central de Venezuela, 2007.

Parallax Inc. 2010. CO2 Gas sensor module. Product Manual.
Pérez M., Martínez D. Diseño de un sistema de comunicación para la transmisión de datos en tiempo real entre tres estaciones meteorológicas ubicadas en el Volcán Antisana y el Instituto Nacional de Meteorología e Hidrología, en Quito, Escuela Politécnica Nacional, Ecuador, 2011.

Rehman, A.; Azafar, A. A., Islam, N. and Ahmed, S. Z. 2014. A review of wireless sensors and networks'applications in agriculture. Computer Standards \& Interfaces. 2(36):263270.

Sonbest. 2018. Soil temperatura and humidity sensor SHT10. Shanghai China.

Sivakumar, M. V. K.; Gommes, R. and Baier, W. 2000. Agrometeorology and sustainable agriculture. Agric. Forest Meteorol. (103):11-26.

Sivakumar, M. V. K.; Roy, P. S.; Harmsen, K. and Saha, S. K. 2004. Satellite remote sensing and gis applications in agricultural meteorology. In: satellite remote sensing and GIS applications in meteorology. Sivakumar, M. V. K.; Roy, P. S.; Harmsen, K. and Saha, S. K. (Eds.) World Meteorological Organization. Dehra Dun, India.427 p.

Torres-Ruiz, E. 2006. $2^{\circ} \quad$ (Ed.). Agrometeorología. México, D. F. Ed. Trillas. $156 \mathrm{p}$.

World Meteorological Organization. 2012. 2008 ed. Guide to Meteorological Instruments and Methods of Observation. WMO No. 8. Geneva. $716 \mathrm{p}$. 